\title{
Seasonal Mood and Behavioral Changes for Japanese Residents in the United Kingdom
}

\author{
Yumiko Kurata $^{1}$, Yumiko Nomura ${ }^{2}$ \\ ${ }^{1}$ Graduate School of Human Sciences, Waseda University, Saitama, Japan \\ ${ }^{2}$ Faculty of Human Sciences, Waseda University, Saitama, Japan \\ Email: kurata@asagi.waseda.jp
}

Received July $10^{\text {th }}, 2012$; revised August $10^{\text {th }}, 2012$; accepted September $5^{\text {th }}, 2012$

\begin{abstract}
The aim of this study was to investigate seasonal changes in mood and behavior for Japanese residents in UK A questionnaire survey was conducted with Japanese residents in the UK $(n=100)$ who participated both a combination winter and summer research. First, a longitudinal study comparing two surveys - one in summer and another in winter-was carried out to determine how the level of seasonal changes influenced depression among Japanese living in the UK. Then, we examined seasonal changes in mood and behavior over a 12-month period based on the degree of seasonal dependence. Paired $t$-tests on Global Seasonality Score (GSS score) and the Center for Epidemiologic Studies Depression Scale (CES-D) scores by winter and summer demonstrated that each score had a significant seasonal difference; individual scores were higher in winter than in summer. We examined the difference between high seasonality group, medium seasonal group, and non-seasonal group, regarding to the winter CES-D and summer CES-D scores. The ANOVA revealed a significant difference on the winter score (Winter: $F(2,97)=4.62$, $p<.01$, Summer: $\mathrm{F}(2,97)=3.24, p<.05)$. Although we did not find any interaction between seasonal change and season, the main effect was significant for season. The results showed fluctuations in which mood, social activity, and sleep all declined during the winter and then improved during the summer. It indicated that depressive symptoms among Japanese living in the UK fluctuate due to seasonality; over a period of 12 months, their mood and behavior declined during winter and improved during summer. As described, Japanese living in the UK experience environmental changes due to seasonality. This suggests that the environmental factor called seasonal change can partly explain why Japanese living in the UK suffer from mental and physical disorders. Mental health measures specific to the local environment are necessary to support individuals to adapt to and live under an environment different from home country.
\end{abstract}

Keywords: Seasonality; Seasonal Change; Depression

\section{Introduction}

Seasonal affective disorder (SAD) is a syndrome characterized by recurrent depression that occurs annually at the same time each year (Rosenthal, Sack, Gilin, Lewy, Goodwin, Davenport, Mueller, Newsome, \& Wehr, 1984). Typically, episodes begin in the fall or winter and remit in the spring (Rosen, Targum, Terman, Bryant, Hofman, Kasper, Hamovit, Decherty, Welch, \& Rosenthal, 1990). Rates of winter SAD and sub-syndromal winter SAD (S-SAD) were significantly higher at the more northern latitudes (Rosen, Targum, Terman, Bryant, Hofman, Kasper, Hamovit, Decherty, Welch, \& Rosenthal, 1990; Magnusson \& Stefansson, 1993; Magnusson \& Axelsson, 1993).

An analysis of seasonal changes in mood, emotion, sleep, and activity levels of people in four regions in Japan with varying latitudes showed that the fluctuations were large in the high-latitude region (Shirakawa, Okawa, Uchiyama, Oguri, Kosaka, Mishima, Inoue, \& Kamei, 1993). Seasonal changes in behavior occur in Japan, but at a lower frequency and with a different profile than in the US or Europe (Ozaki, Ono, Ito, \& Rosenthal, 1995). Seasonal depression peaked in three groups as indigenous white, non-indigenous Asian and Asian-British, with the incidence of winter depression being highest in the Asian group (Suhail \& Cochrane, 1997). Then, since Asian group showed significant seasonal variation in depression, with a greater number of depressive episodes in winter, gender and ethnicity may be major risk factors in winter low mood (Suhail \& Cochrane, 1998). Japanese participants staying in Stockholm showed more mental and somatic depressive symptoms in the winter than in the summer. And the Beck Depression Inventory (BDI) score was higher in participants who had lived in Stockholm for more than 10 years, compared with newcomers who lived in the same city for less than 2 years (Murase, Murase, Kitabatake, Yamauchi, \& Mathé, 1995).

On the basis of these factors, effects on mood and emotions could be predicted when Japanese reside over a long period of time in high-latitude regions with seasonal changes.

Along with the increase in the number of Japanese people living overseas on a long-term basis, the prevalence of mental disorders, mental illnesses, and suicides in such people has increased (Ministry of Foreign Affair of Japan, 2010). In some overseas regions, they may end up living in an environment different from the one home country. Mood and behavior may fluctuate among people in high-latitude regions, where seasonal effects are greater. Exploring the environmental factors affecting mood disorders and mood changes in Japanese people living in a high-latitude region on a long-term basis might provide valuable insight into the pathogenesis, treatment, and prevention of mental illness. 
Therefore, we conducted a longitudinal study to compare the seasonal changes in summer and winter, aiming to understand the its impact on mood and behavior among Japanese living in the UK.

In Study 1, a longitudinal study comparing two surveys - one in summer and another in winter-was carried out to determine how the level of seasonal change influenced depression among Japanese living in the UK. In Study 2, we examined seasonal fluctuations in mood and behavior over a 12-month period based on the degree of seasonal dependence.

\section{Material and Methods}

\section{Study 1}

\section{Sample and Procedure}

We distributed the questionnaire to Japanese men and women over the age of 18 who resided in major areas in the UK (total of 100 individuals: 22 men, average age $=40.55, S D=$ 9.64; 78 women, average age $=41.28, S D=7.78$ ) and asked them to respond in both summer and winter. Recruitment was performed through Japanese companies and Japanese cram schools, and survey forms were sent and collected either by post or by mail. Research consent was obtained after providing participants with written information regarding the research purpose, privacy protection policy, and the voluntary nature of participation. The participants were instructed to fill out the questionnaire anonymously.

\section{Scales}

The Seasonal Pattern Assessment Questionnaire (SPAQ) (Rosenthal \& Wehr, 1987) has several parts covering topics such as the patterns of seasonal dependence, degree of seasonal dependence (Global seasonality score: GSS score), degree of difficulty with seasonal fluctuation, and the degree of that difficulty, and also includes questions about hours of sleep by season.

This study used the Japanese version of the SPAQ (Shirakawa et al., 1993), a standard scale used in Japan for the epidemiological study of SAD.

In this study, the GSS score from the Japanese SPAQ was used as an indicator that measures seasonal fluctuations in mood and behavior. GSS score is the central component of the seasonal pattern Assessment Questionnaire (SPAQ). The GSS retrospectively measures seasonal variations on six different items which are sleep, mood, weight, sleep length, social activity and appetite. The GSS are scaled 0 for no seasonality, 1 for mild, 2 for moderate, 3 for marked and 4 for extremely marked seasonal change. The total score is ranging between 0 to 24 . The cut-off level of the GSS are set to 8 - 10 for sub-SAD and $\geq 11$ for SAD (Rosenthal et al., 1987).

The Center for Epidemiologic Studies Depression Scale (CES-D) Japanese version (Shima, Shikano, Kitamura, \& Asai, 1985 ) is a 20 -item instrument with a 4-point rating system and is a translation of the same scale developed by the National Institute of Mental Health (NIMH). These 20 items are intended screen for depression in the general population. CES-D items are rated with regard to frequency: "Rarely or never (less than once a day)", "Some or a little of the time (1 - 2 days)", "Occasionally or a moderate amount of time (3 - 4 days)", and "Most or all of the time (5 - 7 days)".

\section{Statics}

First, in order to examine the seasonal fluctuations in indi- vidual scores, paired $t$-tests were performed on the GSS and CES-D scores to compare winter and summer scores. In addition, $t$-tests were performed to determine gender differences in the GSS and CES-D scores. Next, comparisons using an analysis of variance (ANOVA) were performed to check whether the length of stay affected the GSS and CES-D scores. Length of stay was compared by dividing participants into four groups: less than three years, less than five years, less than ten years, and ten years or more. Subsequently, we checked for differences in depressive symptoms during summer and winter based on seasonal dependence (GSS scores). This was done by creating groups based on the winter GSS score-high group (11 points or higher), medium group (8 to 10 points), and low group (7 points or lower) - and conducting a one-way ANOVA to test the effect on the winter and summer CES-D scores. Later, various comparisons were made using Tukey's method.

Finally, a two-way ANOVA was conducted to test the effect of two factors - seasonal changes (GSS score) and season (summer and winter) - on the CES-D scores in summer and winter. On the basis of their GSS scores, participants were categorized into a low group (lower than 8 points) or high group (8 points or higher).

\section{Study 2}

\section{Sample and Procedure}

A questionnaire survey was administered to 100 Japanese residents in the UK who participated in both the winter and summer research. Study 2 used the GSS scores and the table of seasonal mood and behavior patterns. The Seasonal Pattern Questionnaire covers topics such as seasonal patterns in mood and behavior.

The Seasonal Pattern Questionnaire contained the following ten items: 1) Feel best, 2) Gain most weight, 3) Socialize most, 4) Sleep most, 5) Eat most, 6) Lose most weight, 7) Socialize least, 8) Feel worst, 9) Eat least, 10) Sleep least. These items may refer to a single month, a cluster of months, or no particular month that stands out as extreme on a regular basis.

\section{Statics}

Responses 1 through 5 were given the score of 1 , and responses 6 through 10 were given the score of -1 . To compare those scores, the high, medium, and low groups were created on the basis of the seasonal assessment score (winter GSS score), and $t$-tests were performed.

PASW for Windows version 18.0 was used for statistical analysis. The demographic characteristics of the participants in Studies 1 and 2 are shown in Table 1.

\section{Results and Discussion}

\section{Study 1}

Paired $t$-tests on GSS scores and CES-D scores by winter and summer demonstrated that each score had a significant seasonal difference; individual scores were higher in winter than in summer (Table 2).

There was no significant gender difference in CES-D and GSS scores in summer or winter. Further, a relationship between seasonal dependence, depression, and the period of residence could not be identified in either the summer period or the winter period. 
Table 1.

Demographic features of participants.

\begin{tabular}{|c|c|c|}
\hline Number & $\mathrm{n}$ & $\%$ \\
\hline Total & 100 & 100 \\
\hline Male & 22 & 22.00 \\
\hline Female & 78 & 78.00 \\
\hline Age & $\mathrm{n}$ & $\%$ \\
\hline Under 29 & 7 & 7.00 \\
\hline 30 ’s & 31 & 31.00 \\
\hline 40 's & 49 & 49.00 \\
\hline 50 's & 13 & 13.00 \\
\hline Mean age & years & $S D$ \\
\hline Total & 41.12 & 8.18 \\
\hline Male & 40.55 & 9.64 \\
\hline Female & 41.28 & 7.78 \\
\hline Duration of stay & $\mathrm{n}$ & $\%$ \\
\hline$<3$ years & 27 & 27.00 \\
\hline$<5$ years & 22 & 22.00 \\
\hline$<10$ years & 27 & 27.00 \\
\hline Over 10 years & 24 & 24.00 \\
\hline Mean months of residence & months & $S D$ \\
\hline Total & 88.79 & 86.17 \\
\hline Male & 82.23 & 52.40 \\
\hline Female & 90.64 & 93.71 \\
\hline Marital status & $\mathrm{n}$ & $\%$ \\
\hline Married & 17 & 17.00 \\
\hline Unmarried & 76 & 78.00 \\
\hline$\underline{\text { Others }}$ & 7 & 7.00 \\
\hline Children & $\mathrm{n}$ & $\%$ \\
\hline With children & 61 & 61.00 \\
\hline Without children & 39 & 39.00 \\
\hline Occupation & $\mathrm{n}$ & $\%$ \\
\hline Student & 4 & 4.00 \\
\hline Housewife & 43 & 43.00 \\
\hline Employee (full time) & 38 & 38.00 \\
\hline Employee (part time) & 7 & 7.00 \\
\hline Others & 8 & 8.00 \\
\hline
\end{tabular}

Next, we examined the difference between the high seasonality group, the medium seasonal group, and the non-seasonal group, regarding to the CES-D scores in winter and summer. The ANOVA revealed a significant difference on the winter score. Multiple comparisons using Tukey's method showed a significant difference between the high group and low group during both winter and summer (Winter: $\mathrm{F}(2,97)=4.62, p<.01$, Summer: $\mathrm{F}(2,97)=3.24, p<.05)$ (Figures 1 and 2 ).

In addition, we conducted a two-way ANOVA to test the effect of two factors-seasonal dependence (GSS score) and season (summer and winter) - on the CES-D score in summer and winter. Although we did not find any interaction between seasonal change and season, the main effect was significant at the .1\% level for season (Figure 3).

\section{Study 2}

An analysis to determine whether seasonal fluctuations in mood and behavior differ over a year indicated significant differences in mood during October, November, and July; in weight during January and December; in social activity during April; in sleep during January and February; and in appetite during January and December.

In terms of mood change, results indicated that the high-GSS group tends to feel worse compared to the low-GSS group during October and November, when the summer has ended and changes such as shortened daylight hours and deteriorations in weather are observed. They also indicated that the high-GSS group tends to feel better than the low-GSS group during July. As for changes in weight, the results indicated that it tends to

Table 2.

Paired $t$-test on the GSS score and CES-D.

\begin{tabular}{ccccc}
\hline & \multicolumn{3}{c}{ Mean } & \\
\cline { 2 - 4 } & Summer & Winter & $t$-Value & \\
\hline GSS & 6.63 & 5.73 & 2.84 & ${ }^{* * *}$ \\
CESD & 22.5 & 10.67 & 16.04 & ${ }^{* *}$ \\
\hline
\end{tabular}

Note: ${ }^{* * *} p<.001,{ }^{* * *} p<.01$; GSS: Global seasonality score; CES-D: The Center for Epidemiologic Studies Depression Scale Japanese version.

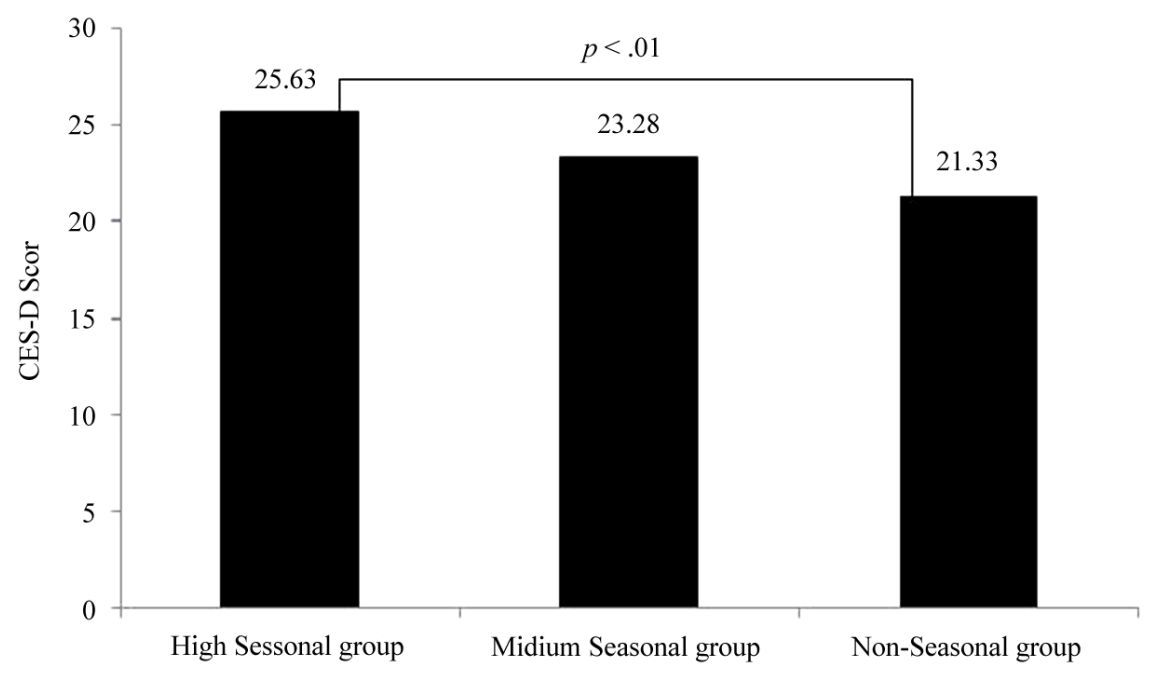

Figure 1.

Mean CES-D for High Seasonal group, Medium Seasonal group and Non-Seasonal group by GSS score (winter). 


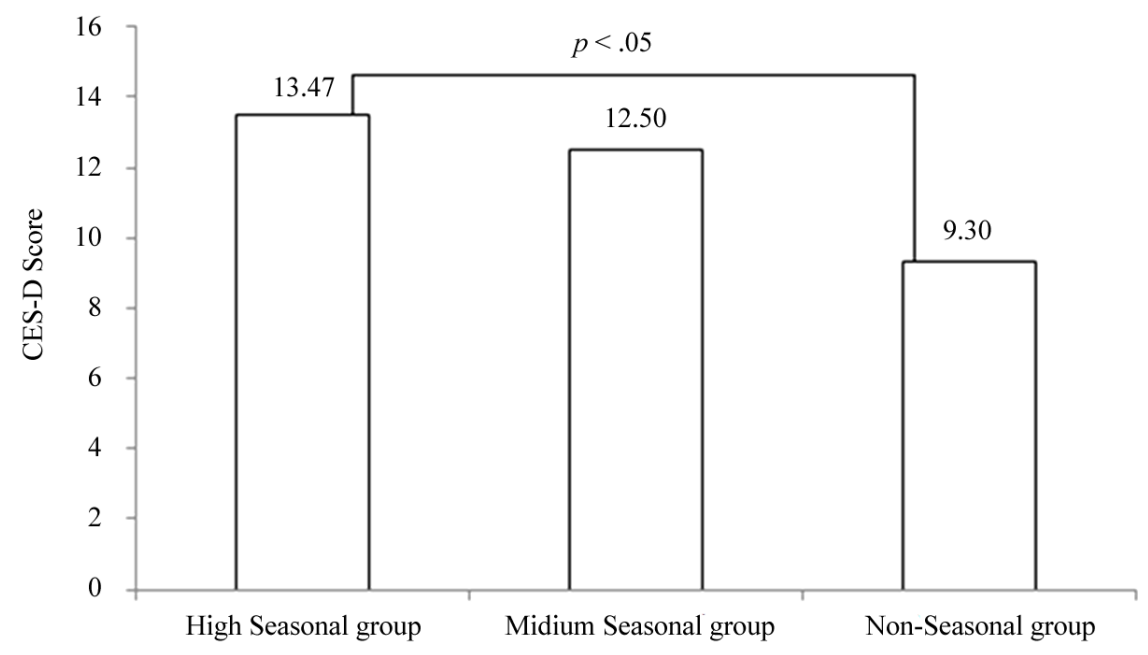

Figure 2.

Mean CES-D for High Seasonal group, Medium Seasonal group and Non-Seasonal group by GSS score (summer).

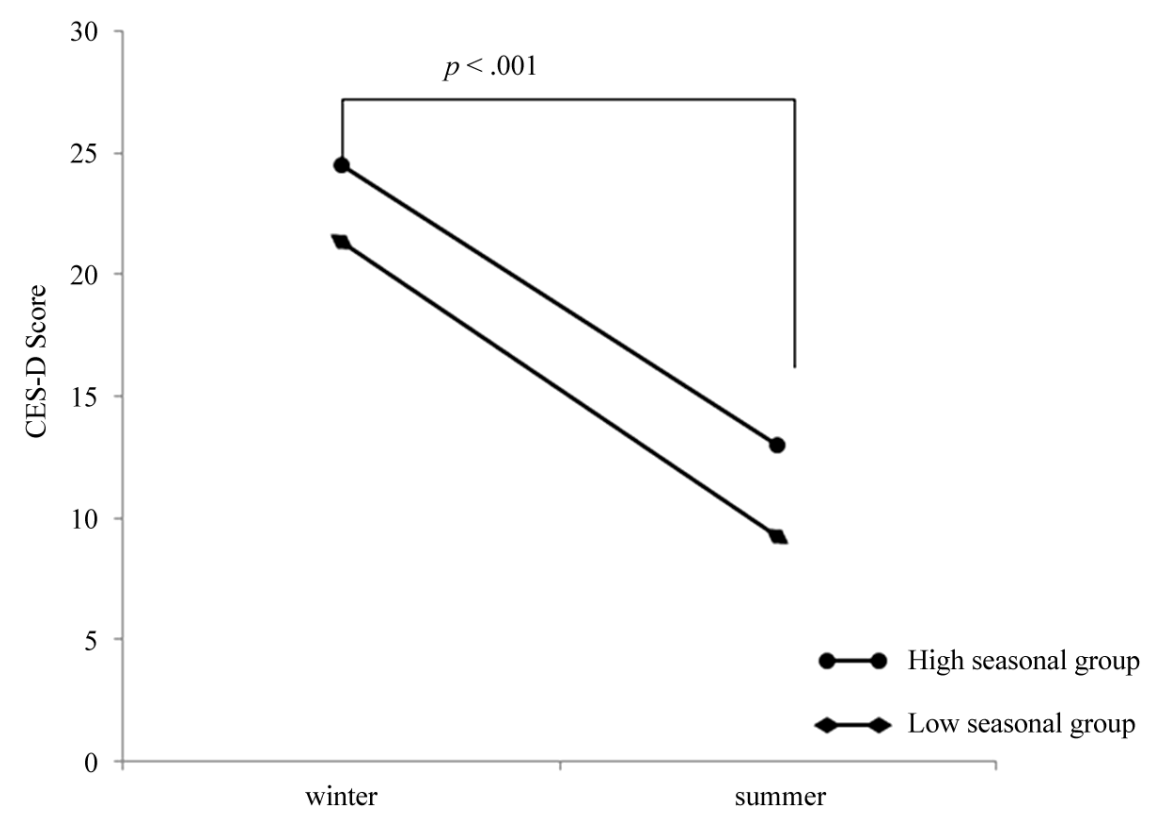

Figure 3.

Graphic representation of the CES-D score in winter and summer by two-factor factorial ANOVA.

increase for the high-GSS group compared to the low-GSS group during January and December. The changes in social activity indicated that they tend to be better for the high-GSS group than the low-GSS group during April. Changes in sleep indicated that the high-GSS group tends to sleep longer than the low-GSS group during January and February. The results indicated that appetite tends to increase during January and December for the high-GSS group. The result of Study 2 is shown in Figures 4 (a)-(e) and Table 3.

\section{Discussion}

This study employed a longitudinal design in which two questionnaire surveys - one in summer, another in winter-were conducted to examine depression. Seasonal change within individuals and aggravation of depression in Japanese residents in the winter was confirmed. In agreement with the findings of other studies, the total CES-D score was significantly higher in winter than in summer. The study also indicated that individuals who are more susceptible to seasonal change are likely to develop more severe depressive symptoms in winter than in summer. In addition, depressive symptoms among Japanese living in the UK were more influenced by the season than the susceptibility to seasonal change.

These results suggested that seasonal change has an important effect on mood among Japanese living in the UK. Further, this study confirmed that depression in Japanese long-term residents of the UK is affected by the season regardless of the length of their residence in the UK.

The results of the present study are not in agreement with previous findings of a study in which participants who had lived in Stockholm for more than 10 years had higher BDI 
Y. KURATA, Y. NOMURA

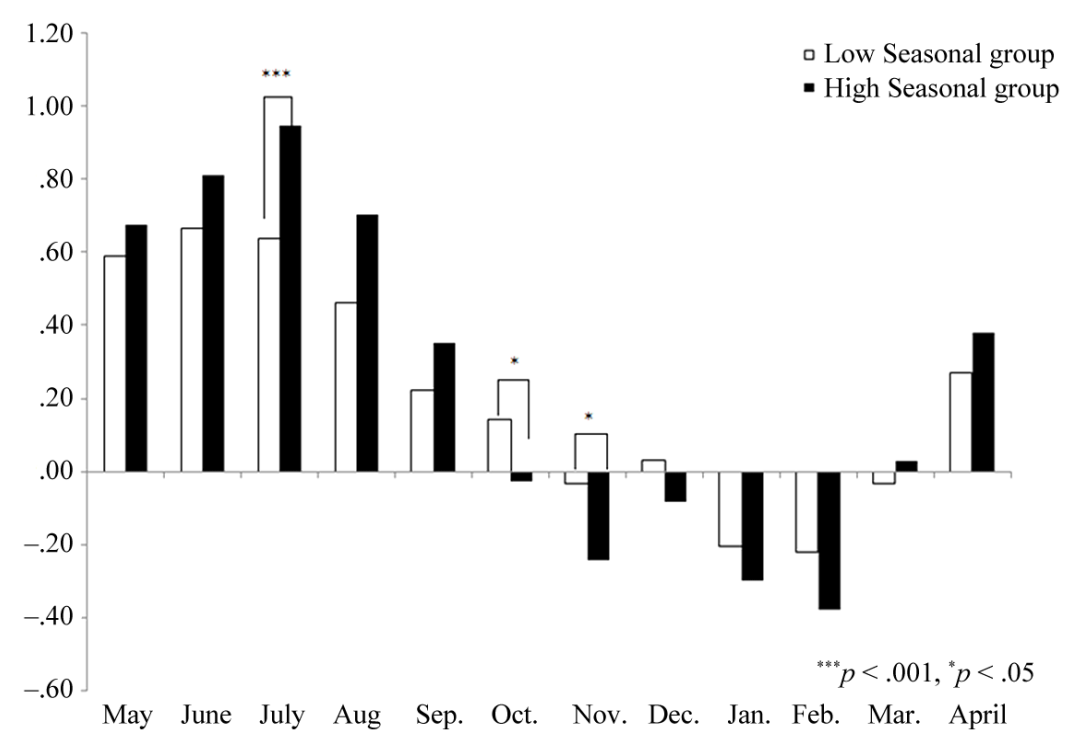

(a)

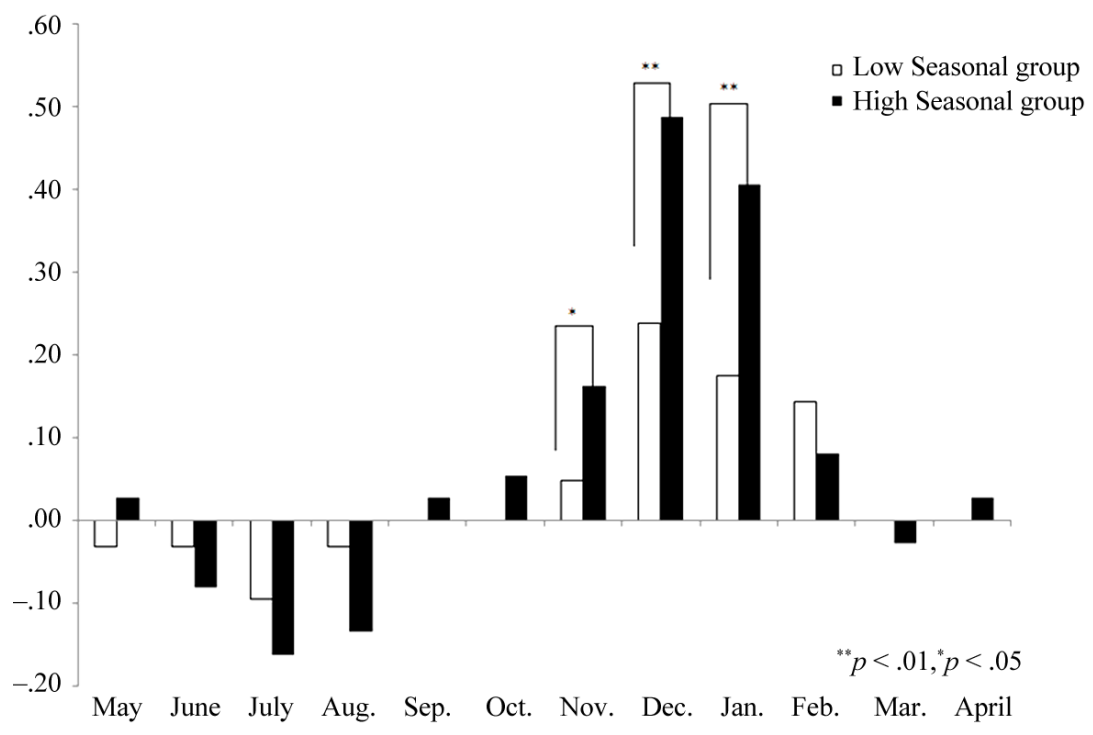

(b)

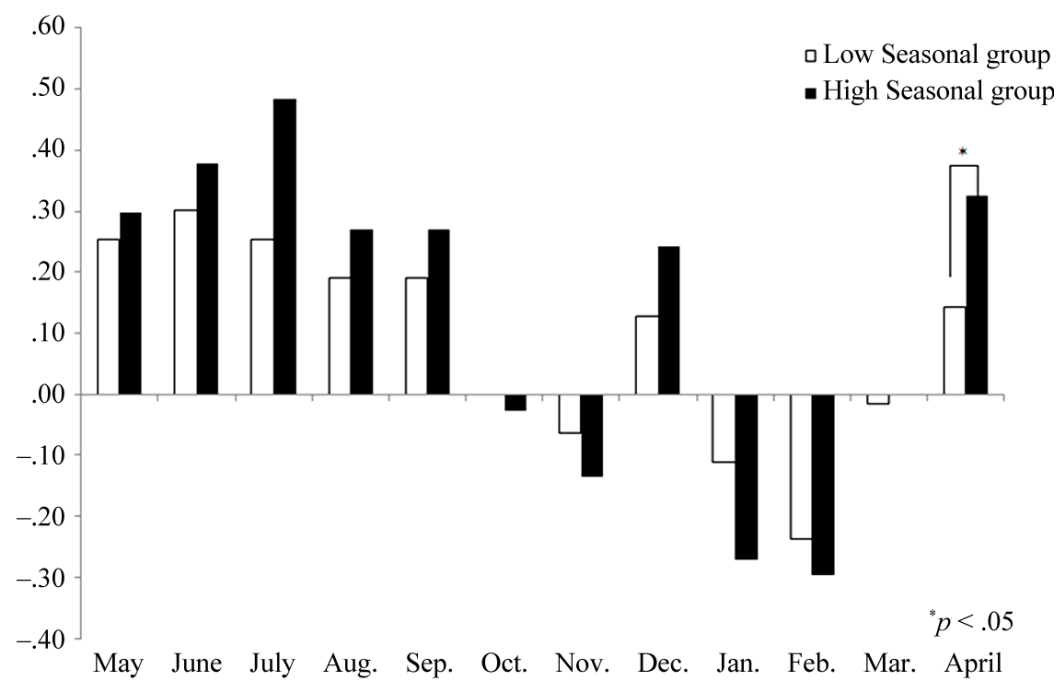

(c) 


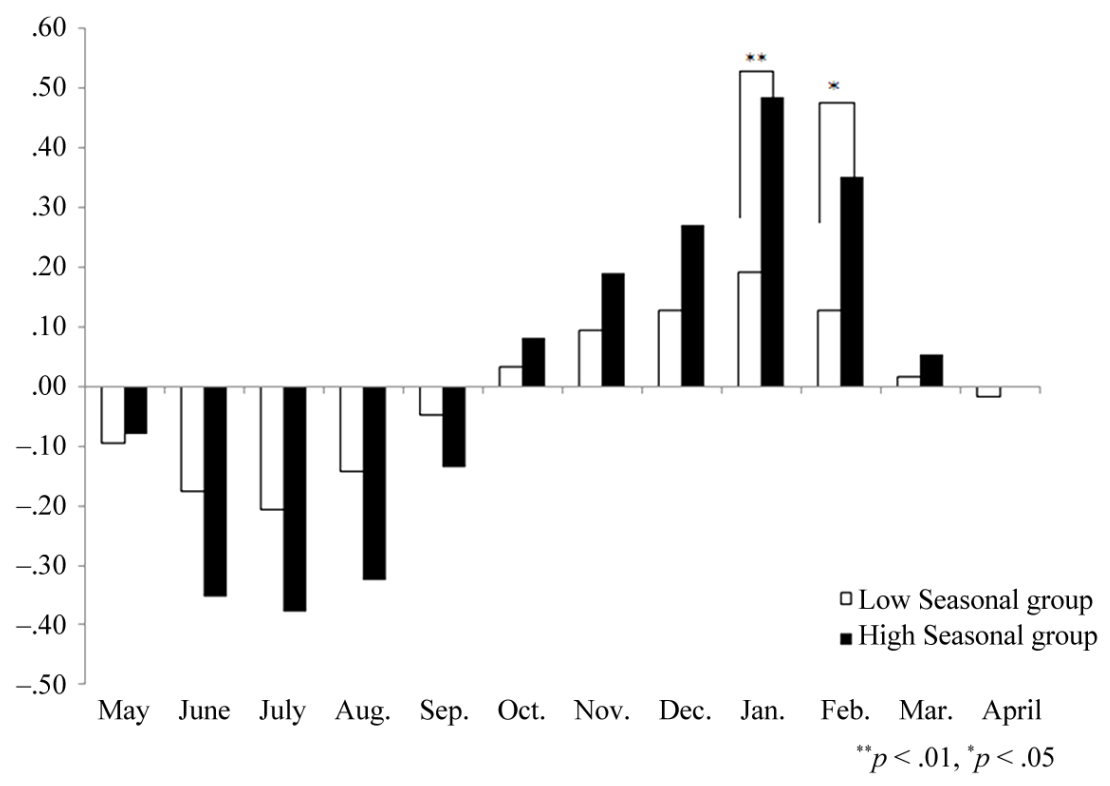

(d)

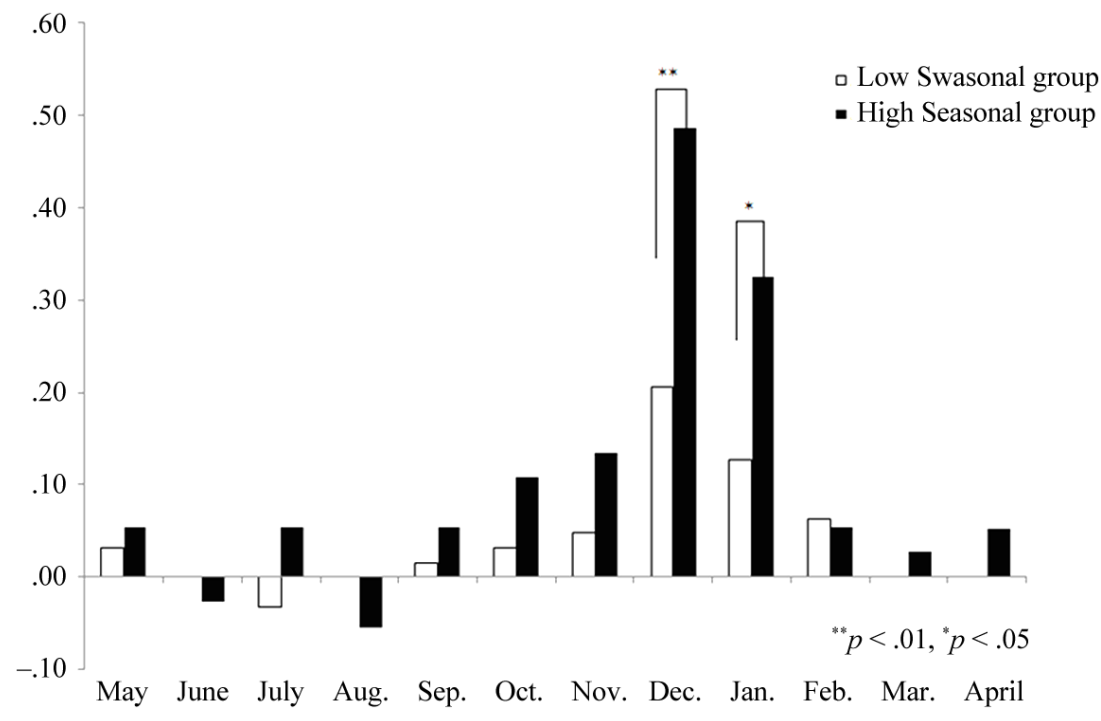

(e)

Figure 4.

Monthly difference of Mood, Weight, Social Activity, Sleep Length and Appetite in participants with high (Global Seasonality Score $\leq 11$ ) or low (Global Seasonality Score $<11$ ) seasonality group. GSS means Global Seasonality Score. (a) Monthly variation of "Mood" between GSS group; (b) Monthly variation of "Weight" between GSS group; (c) Monthly variation of "Social Activity" between GSS group; (d) Monthly variation of "Sleep Length" between GSS group; (e) Monthly variation of "Appetite" between GSS group.

scores than those who had lived in the same city less than 2 years. The difference based on the length of stay may not have arisen in the present study because we used a different scale to measure depressive symptoms and because Stockholm is located at higher latitude than the UK. Since it has been shown that depressive symptoms among Japanese living in the UK are exacerbated during the winter, we believe mental health measures that take seasonal changes into account are necessary regardless of the length of stay.

In this study, no gender differences in depression were found. However, previous research has indicated that women are more vulnerable than men to winter depression (Suhail et al., 1997; Kasper, Wehr, Bartko, \& Rosenthal, 1989; Michalak, Wilkinson, Dowrick, \& Wilkinson, 2001).

The previous findings accord with data showing a lower prevalence of and a lower female-male ratio in seasonal affective disorder in Japan than in Western countries (Shiakamoto, Kamo, Nakadaira, Tamura, \& Takahashi, 1993). Gender may be major risk factor in winter low mood (Suhail et al., 1998). In this study, longitudinal research was conducted using two questionnaire surveys, but the number of participants from both sexes was unequal; hence, gender differences may have been 
Table 3.

Related independent $t$-test on the seasonal pattern score.

\begin{tabular}{|c|c|c|c|c|c|c|}
\hline & $\begin{array}{c}\text { January } \\
t \text {-value }\end{array}$ & $\begin{array}{c}\text { February } \\
t \text {-value }\end{array}$ & $\begin{array}{l}\text { March } \\
t \text {-value }\end{array}$ & $\begin{array}{c}\text { April } \\
t \text {-value }\end{array}$ & $\begin{array}{c}\text { May } \\
t \text {-value }\end{array}$ & $\begin{array}{c}\text { June } \\
t \text {-value }\end{array}$ \\
\hline Mood & 8.50 & 1.69 & .57 & .98 & .88 & 1.48 \\
\hline Weight & $2.6^{* *}$ & .97 & .75 & 1.31 & .96 & .66 \\
\hline Social activity & 1.40 & .56 & 1.80 & $2.05^{*}$ & .43 & .67 \\
\hline Sleep & $2.95^{* *}$ & $2.32 *$ & .59 & .21 & .15 & 1.91 \\
\hline \multirow[t]{2}{*}{ Appetite } & $2.43^{*}$ & .19 & .77 & 1.88 & .51 & .49 \\
\hline & $\begin{array}{c}\text { July } \\
t \text {-value }\end{array}$ & $\begin{array}{l}\text { August } \\
t \text {-value }\end{array}$ & $\begin{array}{c}\text { September } \\
t \text {-value }\end{array}$ & $\begin{array}{l}\text { October } \\
t \text {-value }\end{array}$ & $\begin{array}{c}\text { November } \\
t \text {-value }\end{array}$ & $\begin{array}{c}\text { December } \\
t \text {-value }\end{array}$ \\
\hline$\overline{\text { Mood }}$ & $3.46^{* * *}$ & 2.21 & 1.23 & $1.93^{*}$ & $2.47^{*}$ & 1.39 \\
\hline Weight & .85 & 1.58 & .77 & 1.23 & 1.74 & $2.61^{* *}$ \\
\hline Social activity & 1.86 & .58 & .80 & .33 & .76 & .93 \\
\hline Sleep & 1.71 & 1.78 & 1.38 & .62 & 1.02 & 1.25 \\
\hline Appetite & 1.19 & .82 & 1.08 & 1.56 & 1.56 & $3.03^{* *}$ \\
\hline
\end{tabular}

Note: ${ }^{*} p<.05,{ }^{* *} p<.01,{ }^{* * *} p<.001$.

obscured. It is not conclusive whether the results of this study are specific to the Japanese or due to the gender bias in the sample.

In Study 2, we examined how mood and behavior fluctuate over a period of 12 months among Japanese living in the UK. The results showed fluctuations in which mood, social activity, and sleep all declined during the winter and then improved during the summer. Based on a study of the general population in Japan, Shirakawa et al. (1993) reported that the high-seasonality group experienced larger fluctuations in mood, social life, sleep, and body weight compared to the low-seasonality group. Our study also demonstrated that the high group had larger seasonal fluctuations than the low group did. In particular, the high group experienced a considerable mood decline during the winter, and their mood improvement during the spring and summer was more evident than that of the low group. Similar to studies within Japan, our study showed seasonal fluctuations in mood and behavior. However, while people in both Japan and the UK experienced a decline in mood and behavior in winter, people experienced improvement in summer for the UK and autumn for Japan (Shirakawa et al., 1993; Kamo, Kamo, Nakadaira, \& Sakamoto, 1993). In addition, while a seasonal mood decline was observed twice in Japan, from the rainy season to summer and again during winter, mood declined only during the winter in the UK. Seasonal mood fluctuations differ in the UK and Japan. This is probably because the UK is located at higher latitude than Japan, which would lead to different seasonal changes such as shortened daylight hours and poor weather.

\section{Conclusion}

We found statistically significant changes in mood according to season in Japanese residents of the UK in both the summer and winter studies. In particular, individuals who are more susceptible to seasonal change experience considerably worse depressive symptoms. As described, Japanese living in the UK experience environmental changes due to seasonality. This suggests that the environmental factor called seasonal change can partly explain why Japanese living in the UK suffer from mental and physical disorders. Mental health measures specific to the local environment are necessary to support individuals to adapt to and live under an environment different from home country.

However, a limitation of this study is the skewed ratio: the number of female respondents was higher. Collecting more data by inviting participants without any gender bias will be a key to future research. Hereafter, an increase in the number of examinees should be considered.

Furthermore, although Study 2 demonstrated seasonal mood and behavior fluctuations among Japanese living in the UK, we have not directly compared the findings with those of previous studies. Future studies should investigate differences in seasonal fluctuations in the UK and Japan or those experienced by Japanese living in other countries in order to further clarify the effect of seasonal environment changes.

\section{REFERENCES}

Haggag, A., Eklund, B., Linaker, O., \& Gotestam K. G. (1990). Seasonal mood variation: An epidemiological study in northern Norway. Acta Psychiatrica Scandinavica, 81, 141-145. doi:10.1111/j.1600-0447.1990.tb06467.x

Kamo, T., Kamo, K., Nakadaira, S., \& Sakamoto, K. (1993). A Investigation of seasonality in mood and behavior in normal subjects using the seasonal pattern assessment questionnaire. Japanese Journal of Psychiatry, 35, 837-840.

Kasper, S., Wehr, T. A., Bartko, J. J., \& Rosenthal, N. E. (1989). Epidemiological findings of seasonal changes in mood and behavior: A telephone survey of Montgomery Country, Maryland. Archives of General Psychiatry, 46, 823-833. doi:10.1001/archpsyc.1989.01810090065010

Magnusson, A., \& Stefansson, J. G. (1993). Prevalence of seasonal affective disorder in Iceland. Archives of General Psychiatry, 50, 941946. doi:10.1001/archpsyc.1993.01820240025002

Magnusson, A., \& Axelsson, J. (1993). The prevalence of seasonal affective disorder is low among descendants of Icelandic emigrants in Canada. Archives of General Psychiatry, 50, 947-951. doi:10.1001/archpsyc.1993.01820240031004

Michalak, E. E., Wilkinson, C., Dowrick, C., \& Wilkinson, G. (2001). Seasonal affective disorder: Prevalence, detection and current treatment in North Wales. The British Journal of Psychiatry, 179, 31-34. doi:10.1192/bjp.179.1.31

Ministry of Foreign Affairs of Japan (2010). Annual report of statistics on Japanese nationals overseas. Tokyo: Ministry of Foreign Affairs of Japan.

Ministry of Foreign Affairs of Japan (2010). Kaigai houjin yougo toukei. Tokyo: Ministry of Foreign Affairs of Japan. 
Murase, S., Murase, S., Kitabatake, M., Yamauchi, T., \& Mathé, A. A. (1995). Seasonal mood variation among Japanese residents of Stockholm. Acta Psychiatrica Scandinavica, 92, 51-55.

doi:10.1111/j.1600-0447.1995.tb09542.x

Ozaki, N., Ono, Y., Ito, A., \& Rosenthal, N. E. (1995). Prevalence of seasonal difficulties in mood and behavior among Japanese civil servants. Journal of the American Psychiatric Association, 152, 12251227

Rosen, L. N., Targum, S. D., Terman, M., Bryant, M. J., Hofman, H., Kasper, S. F., Hamovit, J. R., Decherty, J. P., Welch, B, \& Rosenthal, N. E. (1990). Prevalence of seasonal affective disorder at four latitudes. Psychiatry Research, 31, 131-144.

doi:10.1016/0165-1781(90)90116-M

Rosenthal, N. E., Sack, D. A., Gillin, J. C., Lewy, A. J., Goodwin, F. K., Davenport, Y., Mueller, P. S., Newsome, D. A., \& Wehr, T. A. (1984). Seasonal affective disorder. Acta Psychiatrica Scandinavica, 41, 7280. doi:10.1001/archpsyc. 1984.01790120076010

Rosenthal, N. E., \& Wehr, T. A. (1987). Seasonal affective disorders. Psychiatric Annals, 17, 670-674.
Sakamoto, K., Kamo, T., Nakadaira, S., Tamura, A., \& Takahashi, K. (1993). A nationwide survey of seasonal affective disorder at 53 outpatient university clinics in Japan. Acta Psychiatrica Scandinavica, 87, 258-265. doi:10.1111/j.1600-0447.1993.tb03368.x

Shima, S., Shikano, T, Kitamura, T., \& Asai, M. (1985). Seishinigaku. New Self-Rating Scales for Depression, 27, 717-723.

Shirakawa, S., Okawa, M., Uchiyama, M., Oguri, M., Kosaka, M., Mishima, K., Inoue, H., \& Kamei, K. (1993). Seasonality in the mood and behaviors of a normal population sample in Japan. Journal of Mental Health, 39, 81-93.

Suhail, K. \& Cochrane, R. (1997). Seasonal changes in affective state in samples of Asian and white women. Social Psychiatry and Psychiatric Epidemiology, 32, 149-157

Suhail, K. \& Cochrane, R. (1998). Seasonakl variation in hospital admissions for affective disorders by gender and ethnicity. Social Psychiatry and Psychiatric Epidemiology, 33, 211-217.

doi:10.1007/s001270050045 